\title{
Detection and adsorption of Triclosan on sensors based on PAH/PVS thin-films
}

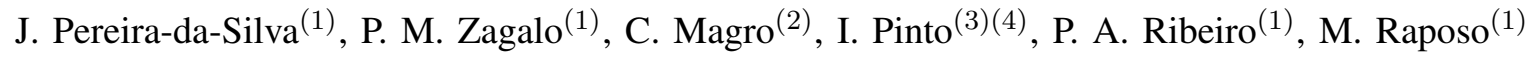 \\ (1) CEFITEC, Departamento de Física, (2) CENSE, Departamento de Ambiente, (3) Centro de Matemática e Aplicações, \\ Faculdade de Ciências e Tecnologia, Universidade Nova de Lisboa, 2829-516 Caparica, Portugal, \\ (4) Instituto Superior de Engenharia de Lisboa \\ \{jvp.silva, p.zagalo, c.magro\}campus.fct.unl.pt, ipintoadm.isel.pt, \{mfr, pfr $\} @$ fct.unl.pt
}

\begin{abstract}
Given the vital role of water in life it is paramount to thrive and succeed in areas that look to clean and prevent further contamination in water bodies. Triclosan (TCS) is present in most of our day-to-day products and if left unchecked it can lead to long-term significant problems due to its many inauspicious effects in both human and animal health. Thus arose the need to develop sensors to detect and possibly remove TCS for aqueous solutions. This work was rooted on this premise and sensors based on LbL polyectrolyte thin-films were developed, such as poly(allylamine hydrochloride) (PAH) and poly(vinylsulfonic acid, sodium salt) (PVS), namely (PAH/PVS) 10 and $(\mathbf{P A H} / \mathrm{PVS}) / \mathrm{PAH}_{10}$. The goal of this work was to analyze the amount of TCS adsorbed onto reused (PAH/PVS $)_{10}$ and $(\mathbf{P A H} / \mathrm{PVS}) / \mathbf{P A H}_{10}$ LbL thin-films when immersed in TCS aqueous solutions with decreasing $\mathrm{pH}$. It was demonstrated that sensors with an outer layer of PAH led to a significantly better TCS molecules adsorption (removal). Additionally, sensors composed of $(\mathbf{P A H} / \mathrm{PVS})_{10}$ presented higher sensibility in discriminating TCS solutions with concentrations between $10^{-5} \mathbf{M}$ and $10^{-8} \mathbf{M}$, using impedance spectroscopy.
\end{abstract}

Index Terms-Triclosan, adsorption, $\mathrm{pH}$, impedance spectroscopy, thin-film, PAH, PVS

\section{INTRODUCTION}

Triclosan (TCS) is present in many pharmaceuticals and personal care products (PPCP) such as toothpastes, soaps and first-aid products [1]. TCS presents a significant drawback given that it has high toxicity levels. Recent works have demonstrated that it can cause alarming effects due to the ability to impair biological responses directly related with thyroid hormone homeostasis and estrogen effects or even the ability to block certain processes that can lead to bacterial resistance, thus resulting in an urgent need to detect and quantify TCS [2, and references therein]. The presence of TCS in the environment has been growing at an accelerated rate inevitably culminating in its deposition in groundwaters, surface waters and soils [3, 4]. Additionally, TCS has been detected in blood, human breast milk and there is evidence that day-to-day products containing TCS promote its accumulation in the human body [2, and references therein].

Recent studies have been well succeeded in using sensors composed of layer-by-layer (LbL) thin-films of polyelectrolytes to detected TCS in water matrices, working as an effective electronic tongue. One of these has used thin-films of (PAH/PAZO) $)_{n}$ (poly (allylamine hydrochloride) (PAH) and poly[1-[4-(3-carboxy-4-hydroxyphenylazo) ben- zene sulfonamido]-1,2ethanediyl, sodium salt] (PAZO)), where $n$ represents the number of bilayers [5]. Additionally, it has been demonstrated that TCS adsorbed amount per unit of area on thin-films of (PAH/PAZO $)_{n}$ is optimized using the positive charged polyelectrolyte $\mathrm{PAH}$ as the outer layer at $\mathrm{pH}$ 6.5 [2]. The adsorption is affected by the solution $\mathrm{pH}$ and the degree of ionization of the last polyelectrolyte of the $\mathrm{LbL}$ film [2]. Therefore, the pKa value of TCS is important for the adsorption of the molecule. TCS $\left(p K_{a}=7.9\right)$ is an ionizable organic chemical that at a typical environmental $\mathrm{pH}(\mathrm{pH} \approx 8)$ exhibits both non-dissociated and dissociated species, which can be estimated through the equations 1 and 2 [2]:

$$
\begin{aligned}
& f_{A}^{N}=\left(1+10^{p H-p K_{a}}\right)^{-1} \\
& f_{I}^{N}=\left(1+10^{p K_{a}-p H}\right)^{-1}
\end{aligned}
$$

Noteworthy, as the $\mathrm{pH}$ increases the TCS solubility also increases [6].

The goal of this work is to analyze the adsorption behavior of TCS with a constant concentration onto reused $(\mathrm{PAH} / \mathrm{PVS})_{10}$ and $(\mathrm{PAH} / \mathrm{PVS})_{10} / \mathrm{PAH} \mathrm{LbL}$ thin-films when immersing in solutions with decreasing $\mathrm{pH}$. Additionally, the impedance spectroscopy technique was used to assess the sensitivity of an array of interdigitated electrodes (IEs) coated with $(\mathrm{PAH} / \mathrm{PVS})_{10}$ for TCS detection in aqueous solutions within the $10^{-15} \mathrm{M}$ to $10^{-5} \mathrm{M}$ concentrations range.

\section{Materials AND Methods}

\section{A. Chemicals and samples preparation}

TCS and the polyelectrolytes were acquired from SigmaAldrich. A stock solution was attained by dissolving 2.895 $\mathrm{mg}$ of TCS into $10 \mathrm{~mL}$ of pure methanol and stored at $4{ }^{\circ} \mathrm{C}$.

The sample solutions for all adsorption tests were prepared by diluting the stock solution in ultra-pure water supplied by a Milli-Q system from Millipore (resistivity of $18 \mathrm{M} \Omega \mathrm{cm}$ ), to achieve a solution of TCS with $10^{-4} \mathrm{M}$ of concentration and containing $1 \%$ methanol. The solutions' $\mathrm{pH}$ were adjusted adding drops of aqueous solutions of $0.10 \mathrm{M}$ sodium hydroxide $(\mathrm{NaOH})$ and hydrocholoric acid $37 \%$ to the TCS solutions. The $\mathrm{pH}$ of the final solutions was measured using a Digital pH Meter ProLab 1000. 
Regarding the impedance spectroscopy, the TCS solutions were prepared by diluting the stock solution in Milli-Q ultrapure water, in order to attain concentrations ranging from $10^{-5}$ $\mathrm{M}$ to $10^{-15} \mathrm{M}$, in increments of $10^{-1} \mathrm{M}$ and containing $1 \%$ methanol.

The thin-films of PAH/PVS were produced using the layerby-layer technique [5]. The solutions of PAH and PVS were prepared with a concentration of $10^{-2} \mathrm{M}$ through the dissolution of the compounds in Milli-Q ultra-pure water.

\section{B. Adsorption experiments}

The ultraviolet-visible (UV-Vis) absorbance spectra of each LbL film was measured, before and after 45 minutes of adsorption, using a UV 2101 PC Scanning Spectrophotometer. After that, the absorbance of the adsorbed TCS on each thinfilm was determined from the difference between both spectra.

Two different LbL thin-films were produced: (PAH/PVS) $)_{10}$ and $(\mathrm{PAH} / \mathrm{PVS})_{10} / \mathrm{PAH}$. Each of them was immersed in several solutions with decreasing $\mathrm{pH}$. The LbL thin-film composed of $(\mathrm{PAH} / \mathrm{PVS})_{10}$ was immersed in solutions with $\mathrm{pH}$ of 2.48, 3.7, 5.68, 6.94, 8.08 and 9.79 and the (PAH/PVS $)_{10} / \mathrm{PAH} \mathrm{LbL}$ thin-film was immersed in solutions with $\mathrm{pH}$ of $3.7,5.68,6.94$, 8.08 and 9.79. The absorbances at $232 \mathrm{~nm}$ and $280 \mathrm{~nm}$ were plotted and compared between each thin-film, because these are the two main UV-Vis absorption bands of TCS [2].

\section{Impedance Spectroscopy (IS) experiments}

Sensors based on (PAH/PVS)10 LbL thin-films deposited on glass supports with inter-digitated gold electrodes were used to detect TCS in aqueous solutions with increasing concentrations, ranging from $10^{-15} \mathrm{M}$ to $10^{-5} \mathrm{M}$. A solution of $0 \mathrm{M}$ was also used as a reference and all measurements were performed at room temperature of $25^{\circ} \mathrm{C}$. The sensors were immersed in the TCS solutions following an increasing order of concentrations, from $0 \mathrm{M}$ up to $10^{-5} \mathrm{M}$. The applied $\mathrm{AC}$ voltage was $1 \mathrm{~V}$ in a frequency range from $1 \mathrm{~Hz}$ up to 1 $\mathrm{MHz}$, using electrical resistance, electrical reactance, electrical capacitance and loss tangent as transducing variables at fixed frequencies. This set of data was attained with a SI 1260 impedance/gain-phase analyzer(Solartron).

\section{Statistical Analysis}

An exploratory analysis of the data was carried out initially, followed by a data modeling phase. In order to analyze the effect of using the PAH or PVS as the outer on TCS adsorption ad consequent UV-vis absorbance, the Student t-test was used for each wavelength, $232 \mathrm{~nm}$ and $280 \mathrm{~nm}$. Regarding the impedance spectroscopy study, principal component analysis (PCA) was used as a multivariate method to reduce the size of data and to obtain a new space of orthogonal components in which different concentration patterns can be identified. The bilateral statistical tests were considered significant when the respective $\mathrm{p}$ values were lower than the significance level of 0.05. Statistical analysis was performed using the SPSS program Statistics ${ }^{\circledR}$ version 22.0. (IBM Corp. Released 2013. IBM SPSS Statistics for Windows, Version 22.0. Armonk, NY: IBM Corp.).

\section{Results}

\section{A. Adsorption experiment}

Figures $1 \mathrm{~A}$ and $1 \mathrm{~B}$ present respectively the absorbances at $232 \mathrm{~nm}$ and $280 \mathrm{~nm}$ of reused sensors based on (PAH/PVS $)_{10}$ and $(\mathrm{PAH} / \mathrm{PVS})_{10} / \mathrm{PAH}$ LbL films after immersion in $10^{-4} \mathrm{M}$ TCS solutions with decreasing $\mathrm{pH}$ of $9.79,8.08,6.94,5.68,3.7$ and 2.48. These absorbances were calculated by subtracting the UV-Vis spectrum after immersion from the initial UV-Vis spectrum of each thin-film.

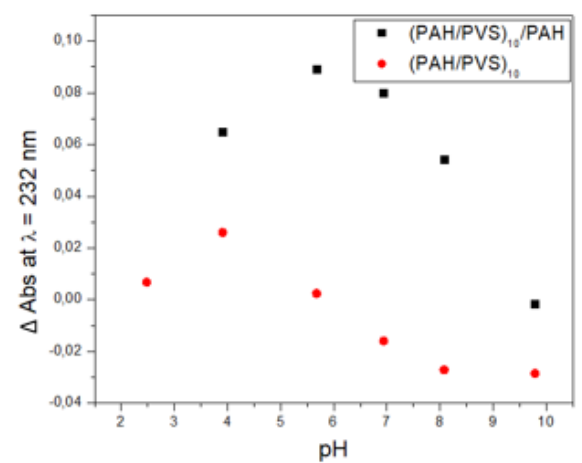

A

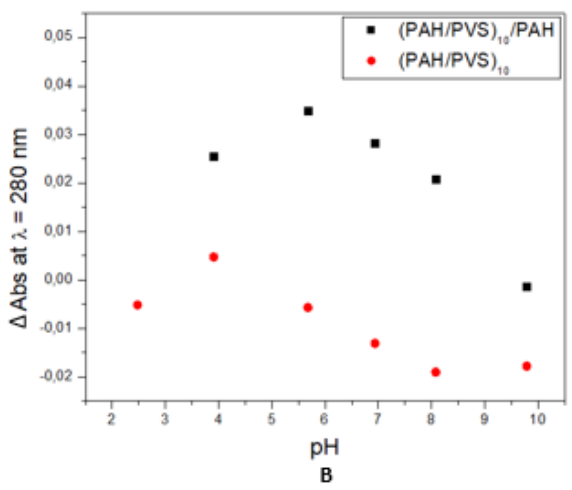

Fig. 1. Absorbances of TCS in the (PAH/PVS $)_{10}$ and (PAH/PVS $)_{10} / \mathrm{PAH}$ thin-films $v s \mathrm{pH}(\mathrm{A})$ at $232 \mathrm{~nm}(\mathrm{~B})$ at $280 \mathrm{~nm}$

In Figures $1 \mathrm{~A}$ and $1 \mathrm{~B}$ it is possible to observe that after the first immersion at $\mathrm{pH} 9.79$, desorption phenomena of the polyelectrolytes PAH/PVS occurred. The (PAH/PVS) 10 and $(\mathrm{PAH} / \mathrm{PVS})_{10} / \mathrm{PAH}$ thin-films revealed a similar adsorption behaviour of TCS. As $\mathrm{pH}$ decreased absorbances tended to increase both at $232 \mathrm{~nm}$ and at $280 \mathrm{~nm}$. The highest absorbances were observed at $\mathrm{pH} 5.68$ for $(\mathrm{PAH} / \mathrm{PVS})_{10} / \mathrm{PAH}$ thin-film and 3.7 for (PAH/PVS $)_{10}$ thin-film. After that, at more acidic $\mathrm{pH}$ the adsorption of TCS tended to decrease at both wavelengths. The (PAH/PVS $)_{10} / \mathrm{PAH}$ thin-film presented significantly higher absorbances values at all analyzed $\mathrm{pH}$ comparing with (PAH/PVS $)_{10}$ thin-film $(p<0.007)$ in both, $232 \mathrm{~nm}$ and $280 \mathrm{~nm}$.

\section{B. Impedance spectroscopy experiment}

Figure 2 shows the dependence of electrical resistance as a function of the frequency. 


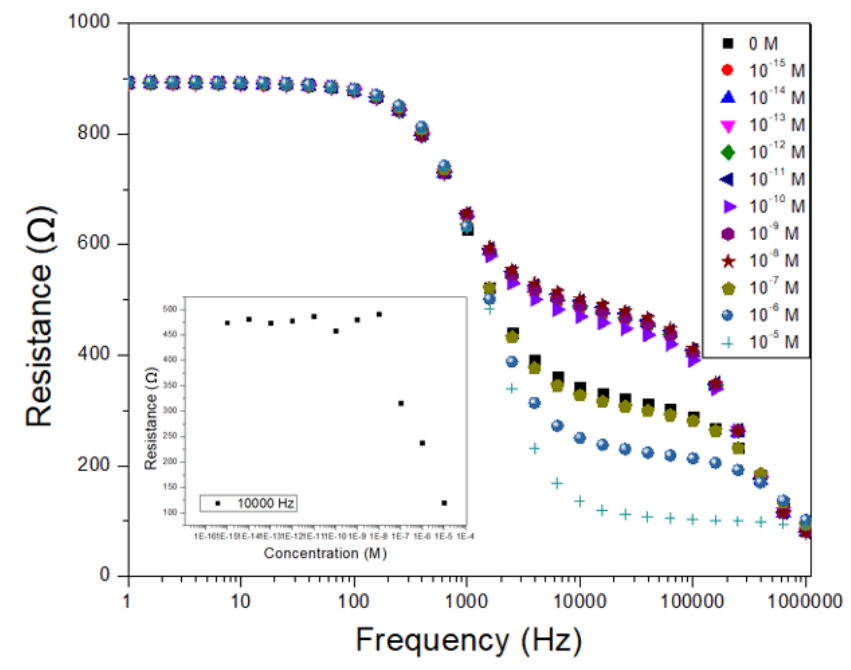

Fig. 2. Resistance spectra of (PAH/PVS $)_{10}$ sensor when immersed in TCS solutions at different frequencies

The figure 2 shows that this sensor is capable of discriminating the different concentrations namely between $1000 \mathrm{~Hz}$ and $25000 \mathrm{~Hz}$ regarding the electrical resistance spectrum. In order to better analyze this particular behaviour, the frequency at $10000 \mathrm{~Hz}$ was chosen and the graph of electrical resistance was plotted in the inset of figure 2 as functions of concentration.

Through the analysis of the electrical resistance at 10000 $\mathrm{Hz}$, it is possible to observe that as the concentration increases, the resistance tends to decrease, namely after $10^{-8} \mathrm{M}$. On the other hand, the capacitance displays an inverse behaviour given that as concentration increases, this electrical parameter tends to increase at $10000 \mathrm{~Hz}$. The PCA methodology (Figure 3) was implemented in order to analyze the impedance spectra at all frequencies.

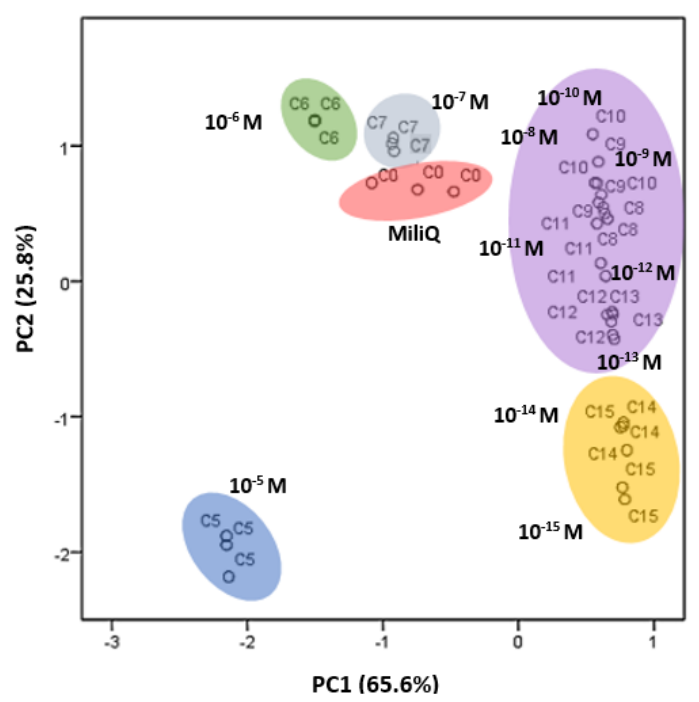

Fig. 3. PCA plot of TCS concentrations $\left(10^{-5} \mathrm{M}\right.$ to $\left.10^{-15} \mathrm{M}\right)$.
It is possible to observe that the TCS concentrations between $10^{-5} \mathrm{M}$ and $10^{-8} \mathrm{M}$ is well discriminated. However, above this range, it is not possible to clearly sort out the rest of TCS concentrations.

\section{DISCUSSION}

A. Successive immersions of PAH/PVS thin-films in TCS solutions with decreasing $\mathrm{pH}$

Figures $1 \mathrm{~A}$ and B show the adsorption behaviour of TCS on $(\mathrm{PAH} / \mathrm{PVS})_{10}$ and $(\mathrm{PAH} / \mathrm{PVS})_{10} / \mathrm{PAH}$ thin-films, respectively. It is possible to observe that the desorption phenomena of $(\mathrm{PAH} / \mathrm{PVS})_{10}$ thin-film are significant at higher $\mathrm{pH}$. It has been demonstrated that the eletrostatic assembly of LbL PAH/PVS thin-films result in poor stability, especially in alkaline solutions [7].

Recent studies demonstrated that the adsorption of TCS onto thin-films composed of polyelectrolytes, is affected by the $\mathrm{pH}$ of TCS solution [2, and references therein]. The $\mathrm{pH}$ affected both the degree of ionization of the last polyelectrolyte in the LbL thin-film and the dissociation of TCS [2, 8]. Using the equations 1 and 2 it was possible to calculate the dissociation fraction of TCS. It begins to dissociate at $\mathrm{pH} 6.14$ and is completely converted into its anionic state ate $\mathrm{pH} 10.14$. Figures $1 \mathrm{~A}$ and $\mathrm{B}$ show that the adsorption capability of TCS on both thin-films is lower at alkaline $\mathrm{pH}$. Regarding $(\mathrm{PAH} / \mathrm{PVS})_{10} / \mathrm{PAH}$ thin-film, this can be explained by the lower amount of positive charges on $\mathrm{PAH}$ layer at alkaline $\mathrm{pH}$ $(p H>p K a)[2,8]$. Thus, the electrostatic attraction between $\mathrm{PAH}$ polyelectrolyte and the deprotonated TCS weakens as the degree of ionization of PAH decreases [2, 8]. Additionally, the hydrogen bonds between the nitrogen of PAH and the phenolic group of TCS, tend also to diminish given that the anionic TCS can not provide hydroxyl hydrogen atoms [9]. Regarding $(\mathrm{PAH} / \mathrm{PVS})_{10}$ thin-film, the amount of negative charges of PVS polyetrolyte is higher at alkaline $\mathrm{pH}$, consequently the electrostatic repulsion overlap the attractive forces [2, and references therein].

As the $\mathrm{pH}$ of TCS solutions decreased the adsorption on both LbL thin-films increased. At $\mathrm{pH} 8.08$ and 6.94, the eletrostatic attraction between the anionic TCS species and PAH tended to increase given that the PAH suface becomes positively charged. At this range of $\mathrm{pH}$ the adsorption of TCS onto (PAH/PVS $)_{10} / \mathrm{PAH}$ thin-film was higher than $(\mathrm{PAH} / \mathrm{PVS})_{10}$ thin-film. The adsorption capability of TCS tends to increase on both $\mathrm{LbL}$ thin-films at $\lambda=232 \mathrm{~nm}$ and $\lambda=280 \mathrm{~nm}$, namely below pH $6.14(p H<p K a)$ [2, and references therein]. At acidic $\mathrm{pH}, \mathrm{TCS}$ is nearly non-dissociated, hence an improved adsorption capacity was observed due to the increase of attractive interactions on the $\mathrm{PAH}$ layer (H-bond formation and hydrophobic interaction) [2, and references therein]. In addition, since protonated TCS molecules are more hydrophobic than the deprotonated anions, increased adsorption is likely to occur on the PAH and PVS layers at lower $\mathrm{pH}[10,11]$. It is important to note that the highest absorbances of TCS onto (PAH/PVS) ${ }_{10} / \mathrm{PAH}$ and $(\mathrm{PAH} / \mathrm{PVS})_{10}$ both at $232 \mathrm{~nm}$ and $280 \mathrm{~nm}$ were at $\mathrm{pH} 5.68$ 
and 3.7, respectively. The highest absorbance on (PAH/PVS $)_{10}$ thin-film was observed at a lower $\mathrm{pH}$. This is probably related to the fact that the sulfonic group of PVS has a low pKa and consequently the negative charges tend to increase with $\mathrm{pH}$ [12]. Hence, the electrostatic repulsion between PVS and TCS are predominant at $p H>3.7$.

\section{B. Impedance spectroscopy experiment}

For the impedance spectroscopy experiment, only the $(\mathrm{PAH} / \mathrm{PVS})_{10}$ thin-film sensor was assessed given that there is less adsorption of TCS than with the (PAH/PVS $)_{10} / \mathrm{PAH}$ thinfilm sensor. Thus, it is possible to deduce that (PAH/PVS $)_{10}$ sensor is more easily reused.

The analysis of the electrical resistance spectrum in the inset of figure 2 reveals that at $10000 \mathrm{~Hz}$ as the concentration increased, the resistance decreased, namely between $10^{-5} \mathrm{M}$ and $10^{-8} \mathrm{M}$ of TCS concentration. This could explained by the gradual increasing of TCS anions in solution. For current to flow, ions must be present in solution to carry the charge from one electrode to another. Increasing the number of ions in solution will increase the amount of charge that can be carried between electrodes and will increase the conductivity. The PCA graph depicted in Figure 3 support the claim that $(\mathrm{PAH} / \mathrm{PVS})_{10}$ thin-film sensor has an acceptable sensitivity in discriminating the concentrations between $10^{-5}$ $\mathrm{M}$ and $10^{-8} \mathrm{M}$ of the TCS solutions. However, below this range of concentrations, it is not possible to sort out lower concentrations, meaning that the (PAH/PVS $)_{10}$ sensor presents a threshold for TCS detection.

\section{CONCLUSIONS}

It has been demonstrated that TCS adsorbed significantly more on $(\mathrm{PAH} / \mathrm{PVS})_{10} / \mathrm{PAH}$ than on $(\mathrm{PAH} / \mathrm{PVS})_{10}$. This phenomenon was affected by the solution $\mathrm{pH}$ and the degree of ionization of the last polyelectrolyte of the thin-film. The highest absorbances of TCS onto (PAH/PVS $)_{10} / \mathrm{PAH}$ and $(\mathrm{PAH} / \mathrm{PVS})_{10}$ were at $\mathrm{pH} 5.68$ and 3.7, respectively. Additionally, the detection of TCS through impedance spectroscopy yielded acceptable results for $(\mathrm{PAH} / \mathrm{PVS})_{10}$ sensors. This sensor showed detection sensitivity for concentrations between $10^{-5} \mathrm{M}$ and $10^{-8} \mathrm{M}$. Thus, the (PAH/PAZO) ${ }_{10} / \mathrm{PAH}$ thin-film is better for the adsorption and possible removal of TCS from solutions. On the other hand, (PAH/PVS $)_{10}$ thin-films are the best option for the production of reusable sensors.

\section{ACKNOWLEDGMENT}

The authors acknowledge the financial support from FEDER, through Programa Operacional Factores de Competitividade-COMPETE and Fundação para a Ciência e a Tecnologia-FCT, by the project PTDC/FISNAN/0909/2014 and for the Portuguese research Grant No. PEst-OE/FIS/UI0068/2011 and UID/FIS/00068/2013. J. Pereira-da-Silva and P.M. Zagalo acknowledge their fellowships PD/BD/142768/2018, PD/BD/142767/2018 from RABBIT Doctoral Programme, respectively. C. Magro acknowledge Fundação para a Ciência e a Tecnologia for her PhD fellowship SFRH/BD/114674/2016.

\section{REFERENCES}

[1] R. D. Jones, H. B. Jampani, J. L. Newman, and A. S. Lee, "Triclosan: A review of effectiveness and safety in health care settings," American Journal of Infection Control, vol. 28, no. 2, pp. 184-196, 2000.

[2] J. Pereira-da-Silva, P. M. Zagalo, G. Magalhães-Mota, P. A. Ribeiro, and M. Raposo, "Adsorption of Triclosan on sensors based on PAH/PAZO thin-films: the effect of $\mathrm{pH}$," Lecture Notes in Bioinformatics, Conference paper, accepted, 2018.

[3] L. W. B. Olaniyan, N. Mkwetshana, and A. I. Okoh, "Triclosan in water, implications for human and environmental health,' SpringerPlus, vol. 5, no. 1, p. 1639, 2016.

[4] M. O. Mendez, E. M. Valdez, E. M. Martinez, M. Saucedo, and B. A. Wilson, "Fate of Triclosan in Irrigated Soil: Degradation in Soil and Translocation into Onion and Tomato," Journal of Environment Quality, vol. 45, no. 3, p. 1029, 2016.

[5] I. Marques, G. Magalhâes-Mota, F. Pires, S. Sério, P. A. Ribeiro, and M. Raposo, "Detection of traces of triclosan in water," Applied Surface Science, vol. 421, pp. 142 147, 2017.

[6] J. Wu, C. Hu, C. Li, Z. Cai, and D. Hu, "Determination of 2,8-dichlorodibenzo-p-dioxin in toothpaste and mouthwash consumer products using GC-MS," Environmental Science and Pollution Research, vol. 22, no. 23, pp. 18 927-18 932, 2015.

[7] F. Ji, Y. Zhang, Y. Geng, Y. Zong, and L. Wang, "Fabrication of covalently linked PAH/PVS layer-bylayer assembled multilayers via a post-photochemical cross-linking strategy," Chemical Research in Chinese Universities, vol. 32, no. 3, pp. 493-498, 2016.

[8] Q. Ferreira, P. J. Gomes, P. A. Ribeiro, N. C. Jones, S. V. Hoffmann, N. J. Mason, O. N. Oliveira, and M. Raposo, "Determination of degree of ionization of poly(allylamine hydrochloride) (PAH) and poly[1-[4-(3carboxy-4 hydroxyphenylazo)benzene sulfonamido]-1,2ethanediyl, sodium salt] (PAZO) in layer-by-layer films using vacuum photoabsorption spectroscopy," Langmuir, vol. 29, no. 1, pp. 448-455, 2013.

[9] C. Lei, Y. Y. Hu, and M. Z. He, "Adsorption characteristics of triclosan from aqueous solution onto cetylpyridinium bromide (CPB) modified zeolites," Chemical Engineering Journal, vol. 219, pp. 361-370, 2013.

[10] Y. Tong, B. K. Mayer, and P. J. McNamara, "Triclosan adsorption using wastewater biosolids-derived biochar," Environmental Science: Water Research and Technology, vol. 2, no. 4, pp. 761-768, 2016.

[11] L. M. G. Abegão, J. Ribeiro, P. A. Ribeiro, and M. Raposo, "Nano-molar deltamethrin sensor based on electrical impedance of PAH/PAZO layer-by-layer sensing films," Sensors, vol. 13, no. 8, pp. 10 167-10 176, 2013.

[12] D. H. Ripin and D. A. Evans, "pKa' s of Nitrogen Acids," Heterocycles, vol. 462, no. 2067, pp. 1-6, 2005. 